\title{
Dapsone: a steroid-sparing drug for refractory immunoglobulin A vasculitis?
}

\author{
Jin-Soon Suh, MD
}

Department of Pediatrics, Bucheon St. Mary's Hospital, College of Medicine, The Catholic University of Korea, Bucheon, Korea

Immunoglobulin A vasculitis (IgAV), formerly called HenochSchönlein purpura, is the most common form of vasculitis in children and characterized by the deposition of IgA-containing immune complexes in the walls of small vessels. The diagnosis is made clinically according to European League Against Rheumatism/Pediatric Rheumatology International Trials Organisation/ Pediatric Rheumatology European Society criteria: purpura or petechia with a lower limb predominance (mandatory) and at least one of four criteria (abdominal pain, histopathology, arthritis or arthralgia, and renal involvement). ${ }^{1)}$ In the majority of pediatric patients with IgAV, the outcome is excellent with spontaneous sign and symptom resolution. Therefore, they do not require specific treatments except for supportive care such as adequate hydration and symptomatic pain relief. However, in more severe cases, specific treatments including immunosuppressive agents and long-term follow-up are needed depending on organ involvement type and degree. The degree of renal involvement is generally considered the most important factor regarding the long-term morbidity of IgAV because a small proportion of children with IgAV nephritis will eventually progress to end-stage renal disease. ${ }^{2)}$ A considerable number of patients experience one or more recurrences regardless of nephropathy status. Although there is no established definition, refractory IgAV may be defined in a subset of IgAV patients who have persistent symptoms including renal involvement or disease recurrence despite of immunosuppressant therapy such as corticosteroids. More importantly, those patients progress to end-stage renal disease more frequently than those who respond well to treatment. ${ }^{3,4)}$ Various trials of several immunosuppressants and anti-inflammatory drugs including dapsone for chronic or refractory IgAV have been reported, mainly in the form of case reports. However, an established treatment for those patients is lacking.

In the recent issue of Clinical and Experimental Pediatrics, Lee et al. ${ }^{5)}$ authored an article entitled "Treatment of refractory Henoch-Schönlein purpura (HSP) with dapsone: a systematic review". In this meta-analysis, the authors reported the treatment responses to dapsone, relapse rates after discontinuation of therapy, and side effects of 26 patients with refractory IgAV
(11 adults, 15 children and adolescents) based on the data of 13 published studies. Almost all patients (24 patients, 92.3\%) responded to dapsone therapy within 7 days. Approximately $65 \%$ of patients experienced relapse after the discontinuation of dapsone and $11.5 \%$ of patients experienced no relapse, while $15.4 \%$ of patients experienced adverse effects of dapsone (arthralgia, rash, and hypersensitivity syndrome). Based on those findings, the authors suggested that dapsone affected refractory IgAV.

Dapsone is an aniline derivative belonging to the group of synthetic sulfones. ${ }^{6}$ Because of its antimycobacterial, antiprotozoal, and anti-inflammatory effects, dapsone has been used as a first-line or adjunctive therapy for chronic inflammatory dermatoses such as leprosy, chronic urticaria, cutaneous lupus erythematosus, and IgA pemphigus. ${ }^{7}$ It has also been used to treat nondermatological diseases such as rheumatoid arthritis, immune thrombocytopenia, stroke, seizure disorder, and glioblastoma. ${ }^{\text {) }}$ Considering that patients with chronic inflammatory conditions frequently need steroids to achieve disease control, dapsone may be a useful and attractive option for those patients, especially pediatric patients who are vulnerable to the side effects of steroids. However, the results of treatment effects for various conditions, even dermatologic diseases, are controversial, and properly controlled trials to prove the effects and safety compared to other drugs are lacking. Therefore, to date, this drug has been recommended as one of several treatment options for refractory chronic inflammatory conditions based on previously reported case reports or literature reviews.

Regarding IgAV, Lee et al. ${ }^{5)}$ also commented that this study included only a small number of IgAV patients and that randomized controlled studies of dapsone and other treatments are lacking. Lee et al..$^{5)}$ did not report on long-term clinical treatment efficacy and safety of dapsone. It is important for clinicians to acknowledge and consider the negative effects of dapsone, including methemoglobinemia, hemolysis, agranulocytosis, various skin eruptions, and neurologic side effects such as peripheral neuropathy with primarily motor function loss, gastrointestinal symptoms, toxic or cholestatic hepatitis, and hypersensitivity syn-

\footnotetext{
Corresponding author: Jin-Soon Suh, MD. Department of Pediatrics, Bucheon St. Mary's Hospital, College of Medicine, The Catholic University of Korea, 327, Sosa-ro, Bucheon, 14647, Korea 
drome; some of these effects are dose-independent. ${ }^{8-10)}$ Dapsone must be used with cautious in patients with glucose-6-phosphate dehydrogenase deficiency, pulmonary diseases, heart failure, severe hepatopathy, and comedication with methemoglobinemiainducing drugs.6) Therefore, before drug initiation, routine evaluations including complete blood count, liver enzymes, urinalysis, and serologic tests for hepatitis, methemoglobinemia, and glucose-6-phosphate dehydrogenase are recommended. $\left.{ }^{6}\right)$ As shown by Lee et al., ${ }^{5)}$ relapse rates after the discontinuation of dapsone were not low. Thus, clinicians and their patients are at risk of experiencing serious side effects such as agranulocytosis or hypersensitivity syndrome when dapsone therapy is used repeatedly.

In conclusion, the paper by Lee et al. ${ }^{5)}$ is of great significance since it introduces a good alternative treatment for refractory IgAV to toxic immunosuppressants for pediatric patients. However, clinicians should be aware of both treatment effects and controversies related to dapsone therapy and consider its benefits and risks. Further studies are needed to prove the safety and effects of dapsone for refractory IgAV.

\section{Conflicts of interest}

No potential conflict of interest relevant to this article was reported.

See the article "Treatment of refractory IgA vasculitis with dapsone: a systematic review" via https://doi.org/10.3345/kjp. 2019.00514.

\section{References}

1. Ozen S, Pistorio A, Iusan SM, Bakkaloglu A, Herlin T, Brik R, et al. EULAR/PRINTO/PRES criteria for Henoch-Schönlein purpura, childhood polyarteritis nodosa, childhood Wegener granulomatosis and childhood Takayasu arteritis: Ankara 2008. Part II: Final classification criteria. Ann Rheum Dis 2010;69:798-806.

2. Trnka P. Henoch-Schönlein purpura in children. J Paediatr Child Health 2013;49:995-1003.

3. Audemard-Verger A, Terrier B, Dechartres A, Chanal J, Amoura Z, Le Gouellec N, et al. Characteristics and management of IgA vasculitis (Henoch-Schönlein) in adults: data from 260 patients included in a French multicenter retrospective survey. Arthritis Rheumatol 2017;69: 1862-70.

4. Crayne CB, Eloseily E, Mannion ML, Azerf SP, Weiser P, Beukelman T, et al. Rituximab treatment for chronic steroid-dependent HenochSchonlein purpura: 8 cases and a review of the literature. Pediatr Rheumatol Online J 2018;16:71.

5. Lee KH, Hong SH, Jun J, Jo Y, Jo W, Choi D, et al. Treatment of refractory Henoch-Schonlein Purpura (HSP) with dapsone: a systematic review. Clin Exp Pediatr 2020 Feb 6 [Epub]. https://doi.org/10.3345/kjp.2019.00514.

6. Wozel G, Blasum C. Dapsone in dermatology and beyond. Arch Dermatol Res 2014;306:103-24.

7. Liang SE, Hoffmann R, Peterson E, Soter NA. Use of dapsone in the treatment of chronic idiopathic and autoimmune urticaria. JAMA Dermatol 2019;155:90-5.

8. Fernandes TRMO, Jesus BN, Barreto TT, Pereira AA. Dapsone-induced agranulocytosis in patients with Hansen's disease. An Bras Dermatol 2017;92:894-7.

9. Choi SY, Hwang HY, Lee JH, Park JS, Jang MS. Severe dapsone hypersensitivity syndrome in a child. Korean J Pediatr 2013;56:260-4.

10. Devarbhavi H, Raj S, Joseph T, Singh R, Patil M. Features and treatment of dapsone-induced hepatitis, based on analysis of 44 cases and literature review. Clin Gastroenterol Hepatol 2017;15:1805-7. 